\title{
Ueber eine neue analytische Behandlungsweise der Brennpunkte.
}

(Von Herrn Siebeck.)

In seiner bekannten Abhandlung über die Brennpunkte der Curven $n^{\text {ter }}$ Classe (Bd. 10 dieses Journals) ist Plücker zu einem Resultate gekommen, welches sich nach einigen Abänderungen kurz so ausdrücken lässt: „Ist bei Annahme eines rechtwinkligen Coordinatensystems $F(u, v, w)=0$ die Gleichung einer Curve $n^{\text {ter }}$ Classe (wobei vorausgesetzt wird, dass die Punktund die Liniencoordinaten durch die Gleichung $x u+y v+w=0$ mit einander verbunden sind), so giebt die Gleichung $F(-1,-i, x+y i)=0$, wo $i=\sqrt{-1}$, die Brennpunkte der Curve." Es gewährt aber besondere Vortheile, wenn man hier, was wie es scheint noch nirgends geschehen ist, $F(-1,-i, x+y i)$ als Function einer Variabeln $z=x+y i$ d. h. als monogene Function betrachtet, denn die Theorie der Brennpunkte algebraischer Curven fällt dadurch lediglich der Algebra binärer Formen anheim. So ergiebt sich z. B. aus der Zerlegung von $F(-1,-i, z)$ in $n$ Factoren, da mit der Gleichung

$$
\left(x+y i-\alpha_{1}-\beta_{1} i\right)\left(x+y i-\alpha_{2}-\beta_{2} i\right) \ldots\left(x+y i-\alpha_{n}-\beta_{n} i\right)=0
$$

zugleich die nachfolgende gilt

$$
\left(x-y i-\alpha_{1}+\beta_{1} i\right)\left(x-y i-\alpha_{2}+\beta_{2} i\right) \ldots\left(x-y i-\alpha_{n}+\beta_{n} i\right)=0,
$$

indem man einen beliebigen Factor der einen dieser beiden Gleichungen zugleich mit einem beliebigen Factor der andern $=0$ setzt (was auf $n^{2}$ verschiedene Arten geschehen kann), nicht allein, dass es, wie Plicker gezeigt hat, im Ganzen $n^{2}$ Brennpunkte giebt, sondern auch dass unter diesen $n$ reell (mit den Coordinaten $\alpha_{1}, \beta_{1}, \alpha_{2}, \beta_{2}$ etc.) und $n(n-1)$ imaginär sind und dass von den letzteren je zwei einander so zugeordnet sind, dass sie in der Normalen liegen, welche man auf der Verbindungslinie zweier reeller Brennpunkte im Mittelpunkte errichten kann. Da somit die imaginären Brennpunkte durch die reellen vollständig bestimmt sind, so wollen wir in dem Nachfolgenden nur die reellen Brennpunkte betrachten.

Es ergiebt sich ferner auf diese Weise, dass $\frac{\partial F(-1,-i, z)}{\partial z}=0$ die Gleichung der $n-1$ Brennpunkte der $1^{\text {ten }}$ Polaren der unendlich entfernten 
Geraden ist, ebenso $\frac{\partial^{2} F(-1,-i, z)}{\partial z^{2}}$ die Gleichung für die $n-2$ Brennpunkte der zweiten Polare etc., $\frac{\partial^{n-1} F(-1,-i, z)}{\partial z^{n-1}}=0$ die Gleichung für den Brennpunkt der $(n-1)^{\text {ten }}$ Polare d. h. für den Mittelpunkt der Curve. Auch ersieht man sofort, dass zwischen diesen Brennpunktgruppen dieselbe Familienzusammengehörigkeit Statt findet, wie zwischen den Curven, zu denen sie gehören, dass insbesondere durch die erste Gruppe, nämlich die Brennpunkte der ursprünglichen Curve, alle folgenden Gruppen vollständig bestimmt sind und von dieser ersten Gruppe in einer lediglich durch monogene Functionen ausgedrückten Abhängigkeit stehen; dass der Mittelpunkt, welcher die letzte Gruppe bildet, der Schwerpunkt einer jeden von den übrigen Gruppen ist; ferner dass zwei Brennpunkte einer gegebenen Curve zusammenfallen, wenn die Discriminante von $F(-1,-i, z)$ verschwindet, etc.

Um von dem Wesen dieser Methode einen deutlicheren Begriff zu geben, wollen wir sie zunächst auf die Kegelschnitte anwenden. Dieselbe Gleichung, welche in der Theorie der Grundgebilde die Bedingung angiebt, unter welcher vier in gerader Linie liegende Punkte harmonisch sind, ist bei Anwendung des Punktcalcüls mit complexen Zahlen der Ausdruck dafür, dass vier in einer Ebene liegende Punkte in einem Kreise liegen und harmonische Punkte dieses Kreises sind (sie enthält nämlich zwei Bestimmungen, wie jede Gleichung zwischen complexen Zahlen). Nun ist aber, wenn zwei binäre Formen zweiter Ordnung $a z^{2}+2 b z+c=0$ und $a^{\prime} z^{2}+2 b^{\prime} z+c^{\prime}=0$ gegeben sind, das Verschwinden der Invariante $a c^{\prime}+a^{\prime} c-2 b b^{\prime}$ die Bedingung dafür, dass die durch jene beiden Formen dargestellten Punktpaare harmonisch liegen. Dieselbe Invariante wird also, wenn wir unter $a, b, c$, z etc. complexe Zahlen verstehen, durch ihr Verschwinden anzeigen, dass die beiden Punktpaare harmonische Punkte eines Kreises sind. Für zwei Kegelschnitte $a_{11} u^{2}+a_{22} v^{2}+a_{33} w^{2}$ $+2 a_{23} v w+2 a_{13} u w+2 a_{12} u v=0$ und $b_{11} u^{2}+b_{k 2} v^{2}+$ etc. $=0$ sind aber die Brennpunkte durch die Gleichungen

$$
\begin{aligned}
& a_{33} z^{2}-2\left(a_{13}+a_{23} i\right) z+a_{11}-a_{22}+2 a_{12} i=0 \\
& b_{33} z^{2}-2\left(b_{13}+b_{23} i\right) z+b_{11}-b_{22}+2 b_{12} i=0
\end{aligned}
$$

bestimmt. Das Verschwinden der obigen Invariante giebt daher die beiden Gleichungen

$$
\begin{gathered}
a_{33} b_{11}+a_{11} b_{33}-2 a_{13} b_{13}=a_{33} b_{22}+a_{22} b_{33}-2 a_{23} b_{23} \\
a_{33} b_{12}+a_{12} b_{33}-a_{23} b_{13}-a_{13} b_{23}=0
\end{gathered}
$$


Hält man dieses Resultat zusammen mit der bekannten Gleichung für denjenigen Kegelschnitt, von dessen Punkten aus nur harmonische Tangenten an die beiden gegebenen Kegelschnitte gezogen werden können, und welcher u. A. auch durch die acht Berührungspunkte der gemeinsamen Tangenten derselben geht, so sieht man sogleich, dass dieser Kegelschnitt, wenn jene beiden Bedingungsgleichungen Statt finden, in einen Kreis übergehen muss. Wir erhalten somit den Satz: Sind die beiden Brenupunktspaare zweier Kegelschnitte harmonische Punkte eines Kreises, so liegen die acht Berührungspunkte der vier gemeinsamen Tangenten beider Kegelschnitte ebenfalls in einem Kreise.

Zu noch interressanteren Resultaten führt der bekannte Satz, nach welchem drei binäre Formen $2^{\text {ten }}$ Grades $f, \varphi, \psi$ drei in Involution stehende Punktpaare geben, wenn $\psi=\lambda f+\mu \psi$ (wo $\lambda$ und $\mu$ constant) und die Doppelpunkte der Involution durch die Hessesche Covariante zweier beliebiger dieser drei Formen dargestellt werden. Sind nämlich $f(u, v, w), \varphi(u, v, w), \psi(u, v, v)$ drei beliebige Kegelschnitte, welche jedoch der Bedingung $\lambda f+\mu \varphi=\psi$ genügen und folglich dieselben vier Geraden berühren, so erhalten wir, da anderntheils $f(-1,-i, z)=0, \varphi(-1,-i, z)=0, \psi(-1,-i, z)=0$ die Gleichungen für die Brennpunte derselben sind, sofort den Satz: Ist ein System von Kegelschnitten einem urd demselben Vierseit einbeschrieben, so stehen die Brennpunktpaare derselben in Kreisinvolution, d. h. es giebt in der Ebene ein festes Punktpaar, welches mit jedem Brennpunktpaare des Systems vier harmonische Punkte eines Kreises bildet. Dieses feste Punktpaar besteht nämlich aus den Doppelpunkten der Kreisinvolution und lässt sich mit Hülfe der Hesseschen Covariante leicht bestimmen.

Vorstehende Bemerkungen mögen genügen, um den Standpunkt anzudeuten, von welchem aus der Verfasser zu den nachstehenden Sätzen gelangt ist, deren Entwickelung, da sie in analytischer Beziehung nichts besonders Bemerkenswerthes darbieten würde, weggelassen worden ist.

I. Lehrsätze über die Curven der Brennpunkte eines Systems von Kegelschnitten, welche demselben Vierseit einbeschrieben sind.

1) Fallen zwei conjugirte Punkte einer Curve dritter Ordnung in die beiden unendlich entfernten imaginären Kreispunkte, so lassen sich unendlich viele Vierseite von der Beschaffenheit construiren, dass jene Curve die Curve der Brennpunkte aller Kegelschnitte ist, welche einem beliebigen dieser Vier- 
seite einbeschrieben sind. Sind nämlich $F_{1}$ und $F_{2}, G_{1}$ und $G_{2}$ zwei beliebige Punktpaare der Curve, welche einander nach demjenigen der drei Beziehungssysteme conjugirt sind, nach welchem die beiden unendlich entfernten imaginären Kreispunkte einander entsprechen, so erhält man eines jener Vierseite, wenn man zwei heliebige Kegelschnitte construirt, welche resp. $F_{1}$ und $F_{2}$, $G_{1}$ und $G_{2}$ zu Brennpunkten haben, und die vier gemeinsamen Tangenten dieser Kegelschnitte zieht. Diese Tangenten bilden nämlich ein solches Vierseit und für jeden demselben Vierseit einbeschriebenen Kegelschnitt liegen die beiden Brennpunkte auf der Curve und sind conjugirle Punkte desselben.

2) Da jede Curve dritter Ordnung so projicirt werden kann, dass zwei conjugirte Punkte in die beiden unendlich entfernten imaginären Kreispunkte fallen, so kann sie auch als Projection einer Brennpunktcurve betrachtet werden. Die rein projectivischen Eigenschaften der Brennpunktcurven sind also allgemeine Eigenschaften aller Curven dritter Ordnung.

3) Entsprechen einander zwei conjugirte Punkte einer Brenpunktcurve nach demselben System, nach welchem die beiden unendlich entfernten imaginären Kreispunkte conjugirt sind, so können sie immer als conjugirte Pole in Bezug auf drei feste gleichseitige Hyperbeln betrachtet werden; und umgekehrt: der Ort der gemeinsamen conjugirten Pole dreier gleichseitiger Hyperbeln ist immer eine Brennpunktcurve (folgt einfach daraus, dass die beiden unendlich entfernten imaginären Kreispunkte conjugirte Pole für alle gleichseitigen Hyperbeln sind).

4) Zieht man von einem Punkte einer Brennpunktcurve vier Tangenten an dieselbe und verbindet man die Berührungspunkte durch sechs gerade Linien, so stehen in dem so entstandenen vollständigen Viereck zwei gegenüberstehende Seiten auf einander normal, nämlich diejenigen, durch welche je zwei zusammengehörige Brennpunkte mit einander verbunden werden.

4) Die Curve der Brennpunkte eines Systems von Kegelschnitten, welche demselben Vierseit einbeschrieben sind, ist vollständig bestimmt durch die Gerade $L$, in welcher die Mittelpunkte der Kegelschnitte liegen und durch die beiden im Eingange erwähnten Doppelpunkte der Kreisinvolution $J$ und $J^{\prime}$, und lässt sich aus diesen Elementen leicht construiren. Man nehme nämlich auf $L$ einen beliebigen Punkt $M$ an, halbire den Winkel $J M J^{\prime}$ und trage auf der Halbirungslinie von $M$ aus nach entgegengesetzten Richtungen Abschnitte $M F$ und $M F^{\prime}$ auf, welche gleich der mittleren Proportionale der Strecken $M J$ und $M J^{\prime}$ sind, so sind $F$ und $F^{\prime}$ zwei conjugirte Punkte der Curve. Als 
specieller Fall ist hier derjenige zu bemerken, in welchem $L$ durch einen der Punkte $J$ und $J^{\prime}$ geht. Die Brennpunktcurve hat dann einen Doppelpunkt, indem nämlich in $J$ oder $J^{\prime}$ zwei conjugirte Punkte vereinigt sind; so dass sich also in dem System der Kegelschnitte ein Kreis befindet.

Beiläufig werde bemerkt, dass

$$
F=\left(x^{2}+y^{2}\right)(\alpha x+\beta y+2 \gamma)+\alpha x-\beta y=0
$$

die Gleichung der Curve ist für ein rechtwinkliges Coordinatensystem, dessen $x$-Achse die Verbindungslinie von $J$ und $J^{\prime}$ und dessen Mittelpunkt die Mitte von $\boldsymbol{J} \boldsymbol{J}^{\prime}$ ist, wobei zugleich angenommen ist, dass die Abscissen von $J$ und $J^{\prime}= \pm 1$ und dass $\alpha x+\beta y+\gamma=0$ die Gleichung von $L$ ist. Ferner hat man

$$
\frac{9}{2} d F=2\left(\alpha^{2}-\beta^{2}-2 \gamma^{2}\right) F+\left(\alpha^{2}+\beta^{2}\right) \varphi,
$$

wo

$$
\varphi=\alpha x^{3}+3 \beta x^{2} y-3 \alpha x y^{2}-\beta y^{3}-3 \alpha x-3 \beta y-2 \gamma,
$$

also ein in Bezug auf $\alpha, \beta, \gamma$ linearer Ausdruck ist. Für die Invarianten $S$ und $\boldsymbol{T}$ hat man die einfachen Ausdrücke

Auch ist

$$
\begin{aligned}
& S=\frac{4}{8 \Gamma}\left(4\left(\alpha^{2}-\beta^{2}-2 \gamma^{2}\right)^{2}-3\left(\alpha^{2}+\beta^{2}\right)^{2}\right), \\
& T=\gamma^{\frac{8}{2}}\left(8\left(\alpha^{2}-\beta^{2}-2 \gamma^{2}\right)^{2}-9\left(\alpha^{2}+\beta^{2}\right)^{2}\right)\left(\alpha^{2}-\beta^{2}-2 \gamma^{2}\right) .
\end{aligned}
$$

$$
\Delta \varphi=6\left(\alpha^{2}+\beta^{2}\right) F .
$$

5) Das Product der Entfernungen des Centrums der Kreisinvolution (welches zugleich der Brennpunkt der dem Kegelschnittsystem zugehörigen Parabel ist) von zwei conjugirten Brennpunkten ist constant, nämlich gleich dem Quadrat der Entfernung der Punkte $J$ und $J^{\prime}$ von diesem Centrum; auch bilden die Verbindungslinien dieses Centrums mit zwei conjugirten Punkten gleiche Winkel mit der Verbindungslinie der Punkte $J$ und $J^{\prime}$. Allgemeiner ist folgender Satz:

Sind $F_{1}$ und $F_{2}, G_{1}$ und $G_{2}, H_{1}$ und $H_{2}$ drei Paar conjugirter Brennpunkte, so ist nur

$$
\frac{H_{1} F_{1} \cdot H_{1} F_{2}}{H_{2} F_{1} \cdot H_{2} F_{2}}=\frac{H_{1} G_{1} \cdot H_{1} G_{2}}{H_{2} G_{1} \cdot H_{2} G_{2}}
$$

und ausserdem die Winkelsumme

$$
G_{1} H_{1} F_{1}+G_{2} H_{1} F_{2}=0
$$

6) Die Enveloppe der Achsen eines Systems von Kegelschnitten, welche demselben Vierseit einbeschrieben sind, ist eine Curve dritter Classe, von 
welcher zwei Brennpunkte mit den Doppelpunkten der mehrfach erwähnten Kreisinvolution zusammenfallen. Der dritte Brennpunkt ist derjenige unendlich entfernte Punkt, welcher in einer zur Geraden der Mittelpünkte normalen Richtung liegt. Je zwei nicht zu ein und demselben Kegelschnitt gehörige Achsen, welche auf einander normal stehen, sind conjugirte Tangenten der Curve und zwar bilden die Durchschnittspunkte derselben mit der Geraden der Kegelschnittmittelpunkte eine Involution. Die Gleichung der Curve ist $\left(u^{2}-w^{2}\right)(\alpha u+\beta v)+\left(u^{2}+v^{2}\right)(\gamma w-\alpha u)=0$.

II. Einige allgemeine Eigenschaften der Brennpunkte algebraischer Curven.

1) Es giebt in der Ebene einer Curve $n^{\text {ter }}$ Classe immer $n$ feste reelle Punkte von der Beschaffenheit, dass, wenn man einen beliebigen Punkt $O$ der Ebene mit diesen Punkten verbindet, die Summe der Winkel, welche diese $n$ Verbindungslinien mit einer beliebigen festen Richtung bilden, sich von der Summe der Winkel, welche die $n$ von $O$ aus an die Curve gelegten Tangenten mit derselben Richtung bilden, nur um ein ganzes Vielfaches von $\pi$ unterscheidet. Jene $n$ festen Punkte sind die reellen Brennpunkte der Curve $n^{\text {ter }}$ Classe.

2) Denkt man sich von dem beliebigen Punkte $O$ aus $n$ Tangenten an die Curve gelegt, welche in beliebiger Ordnung genommen $L_{1}, L_{2}, \ldots L_{n}$ heissen mögen und ausserdem $n$ einander parallele Tangenten von beliebiger Richtung $\boldsymbol{P}_{1}, \boldsymbol{P}_{2}, \ldots \boldsymbol{P}_{n}$, und ist $\boldsymbol{A}_{k}$ der Durchschnittspunkt von $L_{k}$ und $\boldsymbol{P}_{k}$, so bleibt das Product $O A_{1} . O A_{2} \ldots O A_{n}$ bei veränderter Richtung der parallelen Tangenten constant und zwar ist es gleich dem Product der Entfernungen des Punktes $O$ von den $n$ reellen Brennpunkten der Curve.

3) Sind in einer Ebene $n$ beliebige Punkte $P_{1}, P_{2}, \ldots P_{n}$ gegeben, so giebt es immer eine Curve $(n-1)^{\text {ter }}$ Classe, welche die $\frac{n(n-1)}{2}$ Verbindungslinien jener Punkte berührt und zwar so, dass die Berührungspunkte sämmtlich in die Mittelpunkte der Linien fallen. Die $n-1$ reellen Brennpunkte dieser Curve fallen zusammen mit den Brennpunkten der ersten Polaren der unendlich entfernten Geraden in Bezug auf eine beliebige Curve $n^{\text {ter }}$ Classe, welche die Punkte $P_{1} \ldots P_{n}$ zu Brennpunkten hat. Ist z. B. $n=3$, so giebt dies den Satz: Es giebt immer eine Ellipse, welche die Seiten eines gegebenen Dreiecks $P_{1} P_{2} P_{3}$ in ihrer Mitte berührt und die Brennpunkte dieser Ellipse fallen zusammen mit den Brennpunkten des von den sechs Asymptoten 
einer beliebigen Curve dritter Classe, welche $\boldsymbol{P}_{1}, \boldsymbol{P}_{2}, \boldsymbol{P}_{3}$ zu Brennpunkten hat, berührten Kegelschnitts.

4) Bringt man die Gleichung der Brennpunkte $F(-1,-i, z)=0$ einer Curve $n^{\text {ter }}$ Classe auf die Form

$$
z^{n}-n P_{1} z^{n-1}+\frac{n(n-1)}{1.2} P_{2} z^{n-2}-\cdots+(-1)^{n} P_{n}=0,
$$

und setzt $\operatorname{man} P_{k}=p_{k}\left(\cos \pi_{t}+i \sin \pi_{k}\right)$, so ersieht man sofort Folgendes:

a) Es ist $p_{n}$ das Product der Entfernungen des Anfangspunktes der Coordinaten von den $n$ Brennpunkten der Curve; $p_{n-1}$ hat dieselbe Bedeutung für die erste Polare der unendlich entfernten Geraden, $p_{n-2}$ dieselbe Bedeutung für die zweite Polare etc.

b) $\pi_{n}$ ist die Summe der Winkel, welche die $n$ Verbindungslinien des Anfangspunktes der Coordinaten mit den $n$ reellen Brennpunkten mit der $\boldsymbol{X}$-Achse bilden, $\pi_{n-1}$ hat dieselbe Bedeutung für die erste Polare der unendlich entfernten Geraden etc.

Aus dieser Bemerkung in Verbindung mit den vorhergehenden Sätzen lässt sich, indem man den Coordinatenanfangspunkt (Nullpunkt) der Ebene in den Mittelpunkt einer Curve dritter Classe und die Brennpunkte der conischen Polaren der unendlich entfernten Geraden nach \pm 1 verlegt, in Folge der identischen Gleichung

$$
z^{3}-3 z-2 \cos 3 m=(z-2 \cos m)\left(z-2 \cos \left(m+\frac{2 \pi}{3}\right)\right)\left(z-2 \cos \left(m-\frac{2 \pi}{2}\right)\right)
$$

und wenn man berücksichtigt, dass $\cos (x+\alpha i)$ für ein reelles veränderliches $\boldsymbol{x}$ Ausdruck einer Ellipse ist, folgende Construction der Brennpunkte einer beliebigen Curve dritter Classe herleiten:

Sei $O$ der Mittelpunkt der Curve dritter Classe, $I$ und $I^{\prime}$ die Brennpunkte des von den sechs Asymptoten der Curve berührten Kegelschnitts, so lege man an die Curve drei beliebige parallele Tangenten $\boldsymbol{P}_{1}, \boldsymbol{P}_{2}, \boldsymbol{P}_{3}$ und ziehe ausserdem von $O$ aus die Tangenten $L_{1}, L_{2}, L_{3}$; sei nun $A_{k}$ der Durchschnittspunkt von $L_{k}$ und $P_{k}$, und $\alpha_{k}$ der Winkel, welchen $O A_{k}$ mit $O I$ bildet, so beschreibe man mit einem Radius, welcher gleich $\sqrt{\frac{3 A_{1} \cdot O A_{2} \cdot O A_{3}}{2}}$ ist, einen Kreis um $O$ und theile die Peripherie desselben in drei gleiche Theile, so jedoch, dass, wenn $C_{1}, C_{2}, C_{3}$ die betreffenden Theilpunkte sind, für einen derselben die Winkelgleichung

$$
I O C_{1}=\frac{\alpha_{1}+\alpha_{2}+\alpha_{3}}{3}
$$

Journal für Mathematik Bd. LXIV. Heft 2. 
182 Siebeck, über eine neue analytische Behandlungsweise der Brennpunkte.

Statt findet. Hierauf bestimme man die Punkte $D_{l}, D_{2}, D_{3}$ so, dass $D_{k}$ zu $I, I !$ und $C_{k}$ vierter harmonischer Kreispunkt ist, ziehe die Linien $C_{1} D_{1}, C_{2} D_{2}, C_{3} D_{3}$, deren Mittelpunkte $M_{1}, M_{2}, M_{3}$ heissen mögen. Verlängert man dann die Strecken $O M_{1}, O M_{2}, O M_{3}$ um sich selbst über $M_{1}, M_{2}, M_{3}$ hinaus, wodurch man zu den Punkten $F_{1}, F_{2}, F_{3}$ gelangt, so sind letztere die Brennpunkte der Curve dritter Classe. Es mag hier noch bemerkt werden, dass die Linien $O F_{1}, O F_{2}, O F_{3}$ den Flächeninhalt der Ellipse, welche durch $F_{1}, F_{2}, F_{3}$ geht und $O$ zum Mittelpunkte hat, in drei gleiche Theile theilen.

Liegnitz, 1864. 